\title{
In Planta Synthesis of Designer-Length Tobacco Mosaic Virus-Based Nano-Rods That Can Be Used to Fabricate Nano-Wires
}

\author{
Keith Saunders and George P. Lomonossoff* \\ Department of Biological Chemistry, John Innes Centre, Norwich Research Park, Norwich, United Kingdom
}

We have utilized plant-based transient expression to produce tobacco mosaic virus (TMV)-based nano-rods of predetermined lengths. This is achieved by expressing RNAs containing the TMV origin of assembly sequence (OAS) and the sequence of the TMV coat protein either on the same RNA molecule or on two separate constructs. We show that the length of the resulting nano-rods is dependent upon the length of the RNA that possesses the OAS element. By expressing a version of the TMV coat protein that incorporates a metal-binding peptide at its C-terminus in the presence of RNA containing the OAS we have been able to produce nano-rods of predetermined length

OPEN ACCESS

Edited by: Fernando Ponz,

INIA, Spain

Reviewed by:

Markus Sack,

RWTH Aachen University, Germany

James Culver,

University of Maryland, College Park,

United States

*Correspondence:

George P. Lomonossoff

george.lomonossoff@jic.ac.uk

Specialty section

This article was submitted to

Plant Biotechnology,

a section of the journal

Frontiers in Plant Science

Received: 27 April 2017

Accepted: 17 July 2017

Published: 18 August 2017

Citation:

Saunders K and Lomonossoff GP

(2017) In Planta Synthesis

of Designer-Length Tobacco Mosaic Virus-Based Nano-Rods That Can Be

Used to Fabricate Nano-Wires.

Front. Plant Sci. 8:1335.

doi: 10.3389/fpls.2017.01335 that are coated with cobalt-platinum. These nano-rods have the properties of definedlength nano-wires that make them ideal for many developing bionanotechnological processes.

Keywords: tobacco mosaic virus, transient expression, nano-rods, cobalt-platinum binding, nano-wires

\section{INTRODUCTION}

Particles of Tobacco mosaic virus (TMV) consist of a $6.4 \mathrm{~kb}$ molecule of single-stranded RNA encapsidated by approximately 2130 copies of the $17.5 \mathrm{kDa}$ coat protein arranged with helical symmetry. TMV particles are hollow cylinders $300 \mathrm{~nm}$ in length with external and internal diameters of 18 and $4 \mathrm{~nm}$, respectively. TMV is probably the highest yielding plant virus and thus its particles have attracted considerable interest for use in bio- and nanotechnology. For example, peptides have been fused to exposed locations of the coat protein for multi-epitope display and particles have been both genetically and chemically modified. Given their rigid rodshaped morphology, a particular potential use of TMV particles is the production of electrically conductive "nanowires" for the incorporation into nanoscale devices (for reviews of this technology see Lomonossoff and Evans, 2011; Fan et al., 2013).

Though large quantities of TMV rods can be produced by infecting plants with the virus, this approach suffers from a number of disadvantages, the principal of which, from the point of view of the production of nanowires, is that it is difficult to significantly modify the length of the particles without losing infectivity. In addition, there are limitations to the modifications that can be made to the viral coat protein before the productivity of the virus is compromised. As a result there have been several attempts to produce TMV-derived rods without the necessity for virus infection. These approaches have made use of the large amount of information that has been gleaned about the assembly process since the original demonstration that particles can be assembled in vitro through the mixing of the viral RNA and coat protein (Fraenkel-Conrat and Williams, 1955). It is known that assembly is initiated at a single "origin of assembly sequence" (OAS) which, in 
the case of the $\mathrm{U} 1$ or Vulgare strain, is positioned approximately $1 \mathrm{~kb}$ from the $3^{\prime}$ end of the genomic RNA (Zimmern and Wilson, 1976; Zimmern, 1977; Zimmern and Butler, 1977). Initiation of assembly requires the presence of a two-layer disk aggregate (Butler and Klug, 1971), containing 34 coat protein subunits, that interacts with a hairpin structure formed by the OAS. Assembly then proceeds bidirectional, (Butler et al., 1977) with assembly toward the $5^{\prime}$ end of the RNA being considerably faster than that toward the $3^{\prime}$ end (Lomonossoff and Butler, 1979, 1980; Butler, 1984).

Experiments using synthetic RNA molecules transcribed in vitro showed that it is possible to achieve encapsidation of essentially any RNA molecule by the TMV coat protein provided it contains the OAS (Sleat et al., 1986; Turner et al., 1989). It is possible to produce structures more complex than a linear rod by incorporating more than one copy of the OAS on a single RNA molecule resulting in multiple initiation events (Gallie et al., 1987; Eber et al., 2015). However, though highly successful in demonstrating the range of structures that can be assembled from RNA containing the TMV OAS and the TMV coat protein, the in vitro assembly approach has two disadvantages: it is costly to scale up and the coat protein has to be produced separately, usually from TMV-infected plants. This inevitably restricts the range of coat protein mutants that can be used to those which are compatible with the infection cycle. In an attempt to circumvent this limitation, Shire et al. (1990) expressed the TMV coat protein in Escherichia coli. The bacterially expressed protein was unable to assemble with TMV RNA in vitro, a problem which was ascribed to the lack of acetylation of the $\mathrm{N}$-terminal serine preventing the formation of the disk structures necessary to initiate assembly. Hwang et al. (1994) co-expressed the TMV coat protein and a variety of RNA molecules containing the OAS, including full-length TMV genomic RNA, in E. coli. They showed that assembled rods could be detected and, when genomic TMV RNA was used, the purified material could infect tobacco plants. However, the assembly process appeared to be relatively inefficient, with some OAS-containing RNAs being only partially encapsidated. Hwang et al. (1994) hypothesized that this was due to $70 \mathrm{~S}$ ribosomes binding to the $5^{\prime}$ end of the transcribed RNA, thereby interfering with assembly in the faster $3^{\prime}-5^{\prime}$ direction. Using a similar approach, Kadri et al. (2013) expressed the TMV coat protein in the presence or absence of RNA molecules containing the OAS in both bacteria and yeast. They found that TMV-like rods were assembled but that the majority of the encapsidated RNA was of host origin and the length distribution of the rods was similar whether or not OAScontaining RNA was present. Thus this approach was not an efficient way of producing rods of predetermined lengths and the authors speculated on why this was the case. One possible reason for the relatively inefficient encapsidation of the OAScontaining RNA is that the OAS was positioned such that the majority of assembly would have to occur in the relatively slow $5^{\prime}-3^{\prime}$ direction.

Given the relative lack of success obtained previously using heterologous in vivo assembly systems, we decided to examine the possibility of producing defined length rods by transient expression in plants. Since assembly of TMV-like particles has previously been reported in transgenic tobacco plants (Sleat et al., 1986, 1988; Gallie et al., 1987), we rationalized that a plantbased system may be an efficient way of producing rods of predetermined length (Sainsbury and Lomonossoff, 2008). Using the pEAQ-HT vector system (Sainsbury et al., 2009) to express both the TMV coat protein and RNA molecules with the OAS positioned near the $3^{\prime}$ terminus, we demonstrate the efficient production of rods of predetermined length and, furthermore, by the expression of variant coat protein, the generation of nanorods that promote the binding of cobalt-platinum to the exterior surface, thereby producing conducting nano-wires.

\section{MATERIALS AND METHODS}

\section{Molecular Cloning PEAQ-HT-TMV-CP/OAS, PEAQ-HT-TMV-CP(CP9)/OAS, and PEAQ-HT-TMV-CPHis/OAS}

A DNA molecule comprising of the TMV Vulgare strain coat protein sequence (nucleotides 5595-6069; Goelet et al., 1982; GenBank accession number V01408.1), linked to the OAS sequence (nucleotides 5393 - 5578; Goelet et al., 1982) via a linker containing BspEI and SalI restriction sites and containing flanking AgeI and XhoI sites was synthesized (GeneArt), (Supplementary Figure S1A). After digestion with AgeI and XhoI this was ligated into similarly digested pEAQ-HT (Sainsbury et al., 2009) to yield pEAQ-HT-TMV-CP/OAS (Figure 1A). pEAQ-HT-TMV-CP(CP9)/OAS was constructed in a similar manner (Gene Art), (Supplementary Figure S1B) but with the addition of a sequence encoding the peptide CNAGDHANC* (Mao et al., 2004) after the carboxyl terminal amino acid of the TMV coat protein sequence (Figure 1A). Two oligonucleotides, KS 109 and KS 108 (Table 1) were synthesized to clone via PCR with Phusion DNA polymerase (New England Biolabs), a TMV coat protein possessing C-terminal six histidine residues. pEAQ-HT-TMV-CP/OAS was used as a DNA template for this $\mathrm{PCR}$ reaction. The wild-type coat protein sequence in $\mathrm{PEAQ}-H T$ TMV-CP/OAS was then replaced with the modified coat protein sequence via its $A g e I$ and SalI restriction sites (Figure 1A) to yield pEAQ-HT-TMV-CPHis/OAS.

\section{pEAQ-HT-TMV-CP and pEAQ-HT-TMV-CPHis}

The OAS of pEAQ-HT-TMV-CP/OAS and pEAQ-HT-TMVCPHis/OAS were removed by digestion with SalI and XhoI (Supplementary Figure S1A). The resulting DNA was selfligated to create pEAQ-HT-TMV-CP and pEAQ-HT-TMVCPHis.

\section{pEAQ-HT-TMV-CP/NP60/OAS, pBin-KS-mim-OAS-mp del, and pBin_CPMV-2_OAS}

The cowpea mosaic virus (CPMV) VP60 coding region DNA was derived from pBinPS2NT (Liu and Lomonossoff, 2002) by PCR amplification with Phusion DNA polymerase (New England Biolabs) using primers KS 91, and KS 92 (Table 1). The resulting DNA was cloned via the NgoMIV and SalI 


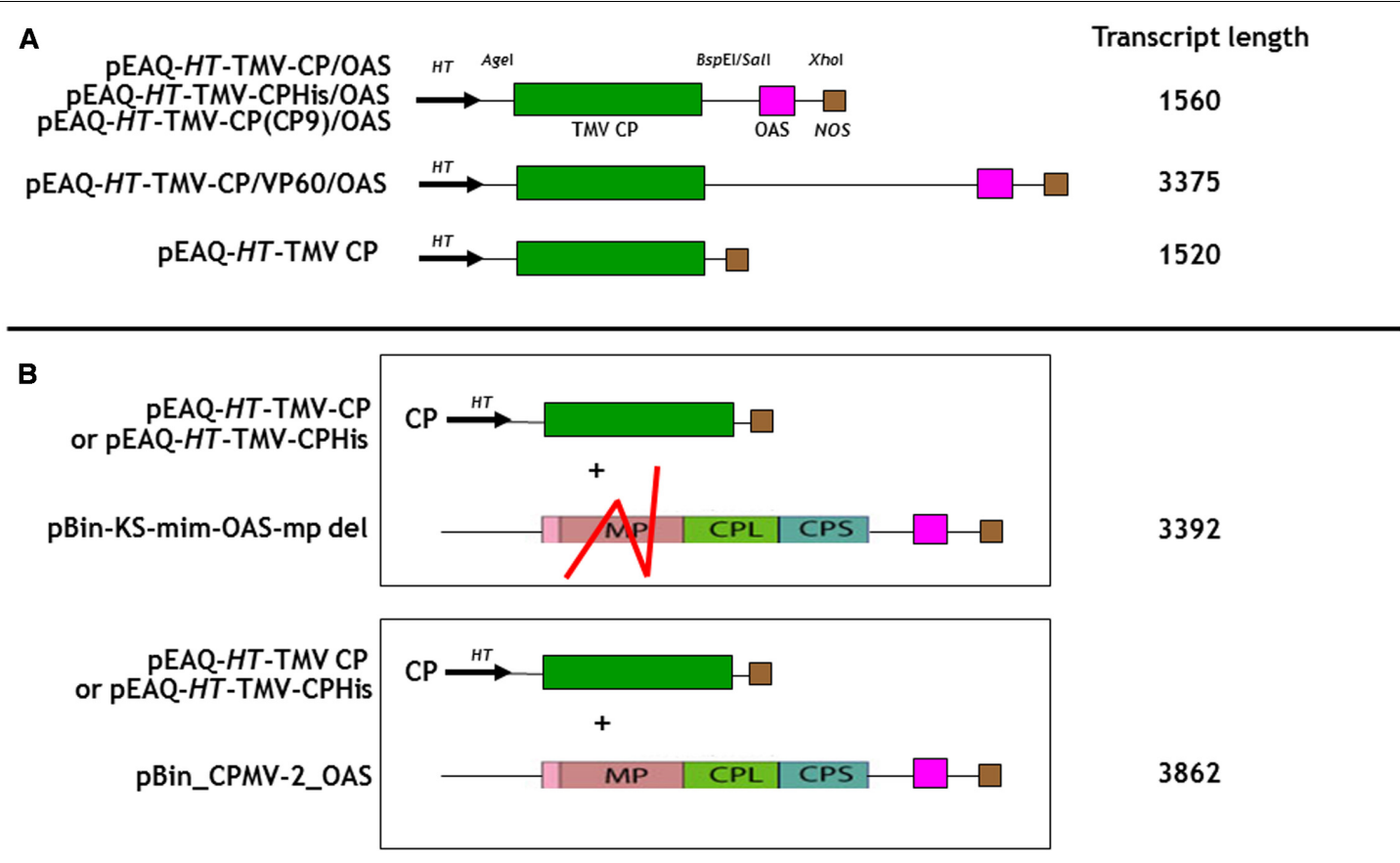

FIGURE 1 | Schematic representation of the infiltrated coat protein gene and OAS element containing gene constructions for (A) In cis and (B) in trans infiltrations. The predicted transcript length for each construct is listed calculated from the start of transcription at the $35 \mathrm{~S}$ promoter to its termination by the nos terminator, Meshcheriakova et al. (2014). TMV CP, green box; the OAS element, pink box and the nos terminator, brown box are indicated. The location of the HT, hyper translatable CPMV 5' UTR sequence (arrowed) is indicated (Sainsbury and Lomonossoff, 2008). Red lines indicate the approximate location of the deleted region of the CPMV movement gene (MP) in pBin-KS-OAS-mp del. CPL and CPS, sequences encoding the CPMV large and small capsid proteins. The locations of relevant restriction sites in the gene constructs are shown.

TABLE 1 | The Sequence of the cloning oligonucleotides.

\begin{tabular}{ll}
\hline Primer & Sequence \\
\hline KS 109 & $5^{\prime}$-AATTCGCGACCGGTTTAAATATGTCTTACAGTATCACTACTCC \\
KS 108 & $5^{\prime}$-TATATATACTCGAGCTATCAGTGATGGTGATGGTGATGAGTTGCAGGACCAGAGGTCC \\
KS 91 & $5^{\prime}$-TATAGCCGGCTAATATGGAGCAAAACTTMTTGGCC \\
KS 92 & $5^{\prime}$-TTAACGTCGACCTTCATTAAGCAGCAGTAGCAGTGTGTC \\
KS 111 & $5^{\prime}$-GTTGAGAGAAGATTACAAACG \\
KS 112 & $5^{\prime}$-TAATACGACTCACTATAGGGCGGTCGAGATCGAAAC
\end{tabular}

restriction sites, (underlined) into BspEI and SalI digested pEAQHT-TMV-CP/OAS to yield pEAQ-HT-TMV-CP/VP60/OAS. DNA corresponding to TMV nucleotides 5420-5546 inclusive (encompassing the OAS, Goelet et al., 1982) was synthesized (GeneArt) and cloned into the movement-deficient CPMV RNA2 vector pBIN-mim-AvrII-mp del (Madi et al., 2015) via its ApaI and AvrII restriction sites to yield pBin-KS-mim-OAS-mp del. BamHI and NotI, unique restriction sites both present in pBin-KS-mim-OAS-mp del and pBin_Mimic_AIV, a derivative of pBinP-EMS-11 (King et al., 2007) were utilized to restore the movement-deficient CPMV property in pBin-KS-mim-OAS-mp del to yield pBin_CPMV-2_OAS.

The DNA sequence of all the modified gene constructs were confirmed by DNA sequencing (Eurofins genomics). Verified pEAQ-HT clones were used to transform Agrobacterium tumefaciens LBA4404 and were subsequently used for plant infiltration experiments (Sainsbury et al., 2014). Infiltration experiments were performed with Agrobacterial cultures at a density of 0.4 OD units/mL in MMA buffer (Sainsbury et al., 2014). Equal volumes of cultures at this density were mix in order to perform co-infiltration experiments.

\section{Isolation of Nano-Rods from Infiltrated Plant Leaves}

Agrobacterium tumefaciens infiltrated leaf tissue was harvested 8 days post infiltration and homogenized in three volumes of extraction buffer (100 mM sodium phosphate, $\mathrm{pH}$ 7.0). After squeezing through Miracloth (Calbiochem), the extracts were clarified by centrifugation at $13,000 \times g$ for $20 \mathrm{~min}$ at $4^{\circ} \mathrm{C}$. To improve the quality of the resulting nano-rods, the supernatant was incubated with one quarter volume of $\mathrm{CHCl}_{3}$ and mixed at this stage and an additional centrifugation step of $13,000 \times g$ for 
$20 \mathrm{~min}$ at $4^{\circ} \mathrm{C}$ was performed. To the resulting supernatant was added one quarter volume of a 20\% (w/v) PEG 6000, $1 \mathrm{M} \mathrm{NaCl}$ solution and the mixture was stirred overnight in a cold room. Protein was sedimented at $13,000 \times g$ for $20 \mathrm{~min}$ at $4^{\circ} \mathrm{C}$ and the pellet was re-suspended in $10 \mathrm{mM}$ sodium phosphate $\mathrm{pH} 7.0$ and further clarified at $27,000 \times g$ for $20 \mathrm{~min}$ at $4^{\circ} \mathrm{C}$. Nano-rods in the resulting supernatant were recovered by centrifugation at $118,700 \times g$ for $3 \mathrm{~h}$ at $4^{\circ} \mathrm{C}$, re-suspended in $10 \mathrm{mM}$ sodium phosphate $\mathrm{pH} 7.0$ and were subjected to centrifugation for $24 \mathrm{~h}$ at $209,627 \times g\left(10^{\circ} \mathrm{C}\right)$ in a $\mathrm{CsCl}$ gradient formed of equal volumes of $42,49,57$, and $65 \%(\mathrm{w} / \mathrm{v}) \mathrm{CsCl}$ in $10 \mathrm{mM}$ sodium phosphate $\mathrm{pH}$ 7.0. After centrifugation, the gradients were illuminated with white light from directly above and photographed. Nano-rodcontaining bands were removed from the $\mathrm{CsCl}$ gradient by side puncture with a syringe and the material was dialysed against $\mathrm{H}_{2} \mathrm{O}$ overnight in a cold room.

\section{Resolution of Coat Protein by Gel Electrophoresis}

Protein samples were denatured in NuPAGE sample buffer (Invitrogen) supplemented with 5\% (v/v) 2-mercaptoethanol and by subsequent heating to $95^{\circ} \mathrm{C}$ for $5 \mathrm{~min}$. Denatured proteins were resolved on $12 \%(\mathrm{w} / \mathrm{v})$ NuPAGE polyacrylamide gels run with MOPS buffer (Invitrogen). See Blue Plus, protein molecular weight markers (Invitrogen) were run on all NuPAGE gels. Instant Blue stain (Expedeon Ltd.) was used to visualize the protein bands. Western blotting to detect either TMV coat protein with an anti-TMV antibody, (AS-72203-2ML, Austral Biologicals, San Ramon, CA, United States) or the six C-terminal histidine residues with a monoclonal antibody prepared to six histidine residues, (Cat 34660, Qiagen) was performed as described by Saunders and Lomonossoff (2015).

\section{Analysis of RNA Encapsidated within Nano-Rods}

Encapsidated RNA was isolated from purified nano-rods by extraction with phenol/ $\mathrm{CHCl}_{3}$ with the addition of 0.2 volume $10 \%(\mathrm{w} / \mathrm{v})$ SDS and 0.1 volume $3 \mathrm{M} \mathrm{Na}$ acetate $\mathrm{pH} 4.8$. After mixing and centrifugation at $16000 \times g$ for $10 \mathrm{~min}$, the RNA in the aqueous phase was recovered following overnight precipitation at $-20^{\circ} \mathrm{C}$ with the addition of 2.5 volume of ethanol and by centrifugation at $16,000 \times g$ for $20 \mathrm{~min}$. RNA pellets were washed with $70 \%(\mathrm{v} / \mathrm{v})$ ethanol and after centrifugation at $16,000 \times g$ for $20 \mathrm{~min}$, the RNA was air-dried and suspended in $20 \mu \mathrm{L} \mathrm{H} \mathrm{H}_{2} \mathrm{O} .5 \mu \mathrm{L}$ of RNA was denatured by the addition of formaldehyde $(0.7 \mathrm{vol})$ and formamide (2 volume), incubated at $65^{\circ} \mathrm{C}$ for $5 \mathrm{~min}$ and subsequently resolved in $1.2 \%(\mathrm{w} / \mathrm{v})$ agarose gel containing $2.2 \mathrm{M}$ formaldehyde. The RNA size marker, catalog number 15623-200 (Invitrogen) was run alongside the extracted RNA samples and the RNA detected under UV light. For northern blot analysis, the RNA resolved in an agarose gel was stained by ethidium bromide and subsequently transferred to a positively charged nylon membrane by overnight capillary transfer in $20 \times \mathrm{SSC}(3 \mathrm{M} \mathrm{NaCl}, 300 \mathrm{mM}$ Na citrate). The RNAs containing the OAS sequence were detected by hybridisation with a complementary 126 nucleotide DIG-labeled RNA probe
(Meltzer et al., 1998) containing the OAS sequence, (nucleotides 5546 to 5420 , Goelet et al., 1982). This was prepared by T7 RNA transcription of a PCR product made using pEAQ-HTTMV CP/OAS as the template DNA with the primers KS 111 and KS 112, (Table 1), the latter possessing the T7 RNA polymerase promoter sequence.

\section{Transmission Electron Microscopy}

Particle preparations were spotted onto carbon-coated copper grids and when necessary were negatively stained with $2 \%(\mathrm{w} / \mathrm{v})$ uranyl acetate. The grids were examined using a Tecnai 20 transmission electron microscope. Nano-rod length of TMV rod-like structures that clearly resembled TMV-like particles, was determined by utilizing AMT camera software version 3.2 (Supplementary Table S1).

\section{Cobalt Platinum Binding}

After gradient purification, a volume of nano-rods were incubated at room temperature with an equal volume of a 1:1:1: solution of $50 \mathrm{mM} \mathrm{CoCl}_{2}, 50 \mathrm{mM} \mathrm{H}_{2} \mathrm{PtCl}_{6}$ and $100 \mathrm{mM}$ $\mathrm{NaBH}_{4}$ for 22 or $69 \mathrm{~h}$ with constant agitation. Nano-rods were spun-washed at $3,000 \times g$ with $1 \mathrm{~mL} \mathrm{H} \mathrm{H}_{2} \mathrm{O}$ in Microcon YM-100 MWCO $100 \mathrm{kDa}$ spin filters (Millipore) prior to electron microscopy.

\section{RESULTS AND DISCUSSION}

\section{Creation of an "In cis" Assembly System}

As a first step to creating defined length nano-rods, we designed a DNA sequence that included the TMV coat protein open reading frame with a TMV OAS element to its $3^{\prime}$ side. This reverses the order of the OAS and coat protein $(\mathrm{CP})$ that is found in TMV U1 genome and was done to ensure that the majority of RNA encapsidation would occur in the fast $3^{\prime}-$ $5^{\prime}$ direction. Upon transcription, this gene construct would direct the synthesis of TMV CP which will subsequently interact with the OAS element to form nano-rods. As both the CP and OAS are present on the same RNA, we term this the in cis approach. This synthetic DNA was cloned into pEAQ-HT (Figure 1A), a plant-based expression vector that produces abundant quantities of highly translatable RNA (Sainsbury et al., 2009) to yield pEAQ-HT-TMV CP/OAS. The predicted length of the RNA transcribed from this construct is 1560 nucleotides, including sequences derived from the pEAQ vector (Meshcheriakova et al., 2014), approximately one quarter of the length of genomic TMV RNA. Subsequently an additional stretch of nucleic acid from the unrelated plant virus, cowpea mosaic virus, was placed between the TMV coat protein and OAS elements, to give construct pEAQ-HT-TMV$\mathrm{CP} / \mathrm{VP} 60 / \mathrm{OAS}$ which is predicted to give a transcript of 3375 nucleotides, approximately twice as long as that from pEAQHT-TMV CP/OAS. As a control a further construct, pEAQ$H T$-TMV CP, was created which was designed to produce RNA encoding the TMV CP but which lacks the OAS. (Figure 1A). 
To determine if nano-rods could be formed from the above constructs, Nicotiana benthamiana leaves were separately infiltrated and extracts were subjected to the standard TMV purification protocol including precipitation with PEG and ultracentrifugation, followed by isopycnic centrifugation on caesium chloride $(\mathrm{CsCl})$ gradients. Material banding at the same density as an authentic TMV control was collected and the $\mathrm{CsCl}$ removed by dialysis. Figure $\mathbf{2 A}$ shows the typical position in a $\mathrm{CsCl}$ gradient of the nano-rods resulting from the infiltration of plant leaves with pEAQ-HT-TMV CP/OAS. NuPAGE gel electrophoresis and Instant Blue staining revealed the presence of a single band of the size expected for TMV coat protein in the nano-rod-containing fractions of the $\mathrm{CsCl}$ gradients in the case of pEAQ-HT-TMV-CP/OAS and pEAQHT-TMV-CP/VP60/OAS (Figure 2B, lanes 1 and 2). The identity of this protein was confirmed by MALDI-TOF analysis of tryptic digests of the material in the bands (data not shown). By contrast, no band corresponding to TMV CP could be seen in extracts prepared from leaves infiltrated with pEAQ-HTTMV CP, a construct that is designed to produce TMV CP but no OAS-containing transcripts. These results indicate that expression of both the TMV CP and the presence of an RNA containing the OAS is essential for the formation of TMV nano-rods and that the buoyant density of such artificial rods in $\mathrm{CsCl}$ is similar to that of native TMV. This indicates that the nano-rods have a similar RNA:protein ratio, and therefore structure, to native TMV. The main difference between the results obtained with pEAQ-HT-TMV-CP/OAS and pEAQ-HTTMV-CP/VP60/OAS was the level of CP detected in latter which was only about $10 \%$ of that for the shorter construct in samples from which equal masses of leaf material was processed.
To confirm that the TMV CP was incorporated into TMVlike rods of defined length the particles were examined by transmission electron microscopy using negative staining. The particles appeared to have identical external and internal diameters to native TMV particles but in both cases were shorter (Figure 3A, panels 1 and 2). The length of a TMV particle is dependent upon the length of its encapsidated genome with one TMV coat protein subunit interacting with three nucleotides. Therefore, the expected length of each type of nano-rod can be calculated from the number of nucleotides in the encapsidated RNA. The length of a representative number of particles was measured from the micrographs in Figure 3A and there was very good agreement between the calculated and measured lengths for each class of nano-rod (Figure 3B, panels 1 and 2), confirming our hypothesis that it should be possible to vary the size of nano-rods by changing the length of the RNA containing the OAS.

\section{Creation of an "In trans" Assembly System}

Although the "in cis" approach described above was capable of producing nano rods of predetermined size it lacks flexibility. The production of the TMV CP from the same RNA that contains the OAS means that there is a minimum length of particle that can be produced and it is possible that encapsidation of the mRNA for the CP may adversely affect its translation. This, and increasing the length of the RNA to be encapsidated, may lead to insufficient CP being produced to completely encapsidate the OAS-containing RNA. We suspect that either or both of these phenomena are the cause of the low yield of nano-rods from pEAQ-HT-TMV-CP/VP60/OAS compared with
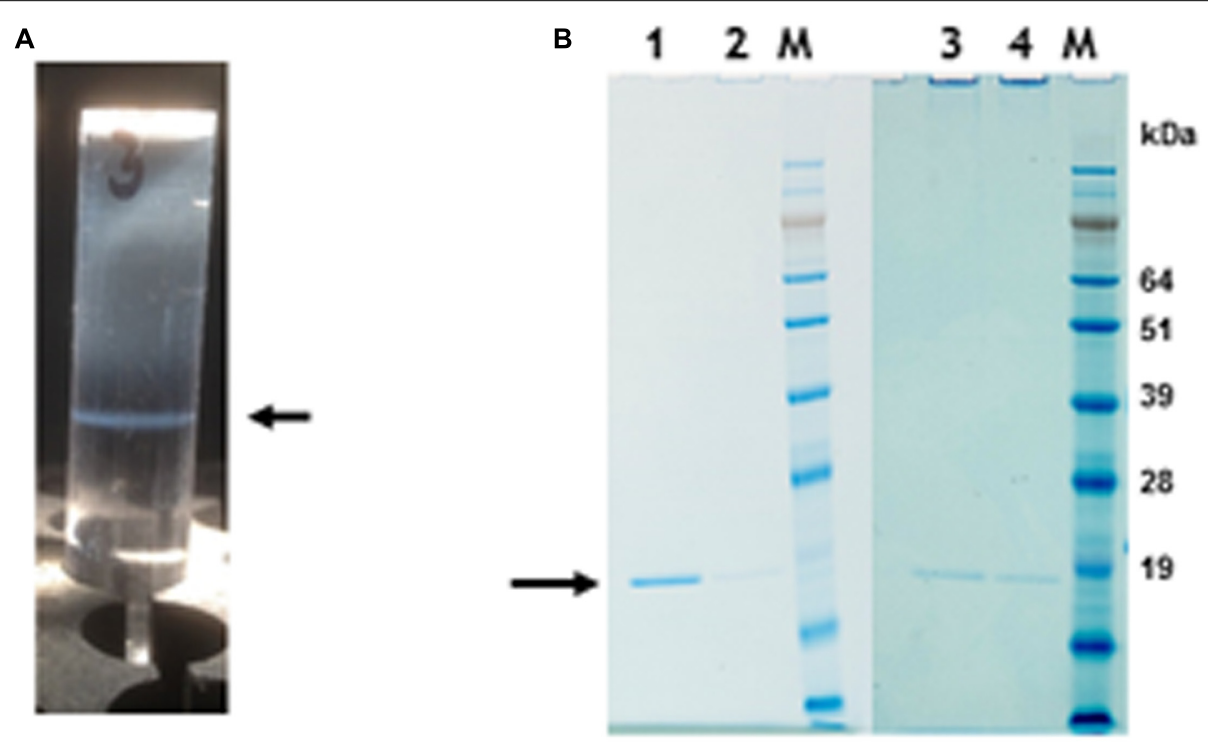

FIGURE 2 | Protein gel analysis and CsCl gradient separation of nano-rods. (A) CsCl gradient resolution of pEAQ-HT-TMV CP/OAS nano-rods. Arrow indicates the position of the nano-rods in CsCl gradient. (B) NuPAGE gel analysis of the coat protein after PEG purification of nano-rods. Lane 1, pEAQ-HT-TMV CP/OAS. Lane 2, pEAQ-HT-TMV CPNP60/OAS. Lane 3, co-infiltration of pEAQ-HT-TMV/CP and pBin-KS-mim-OAS-mp del. Lane 4 co-infiltration of pEAQ-HT-TMV/CP and pBin_CPMV-2_OAS. M, protein marker standards. Arrow indicates the position of TMV CP. Original images are shown in Supplementary Figure S4. 
A
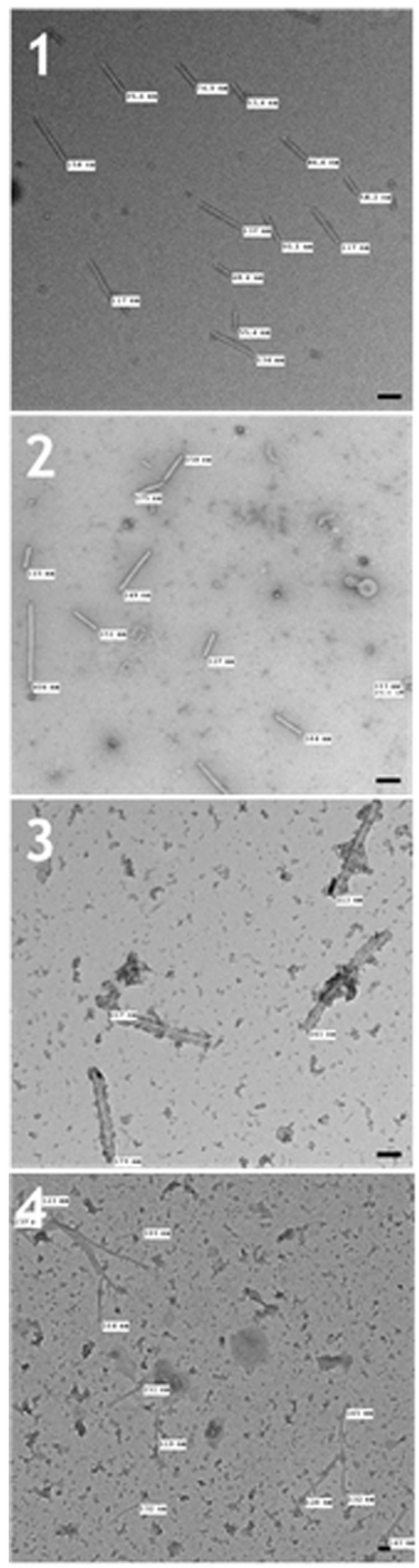

B

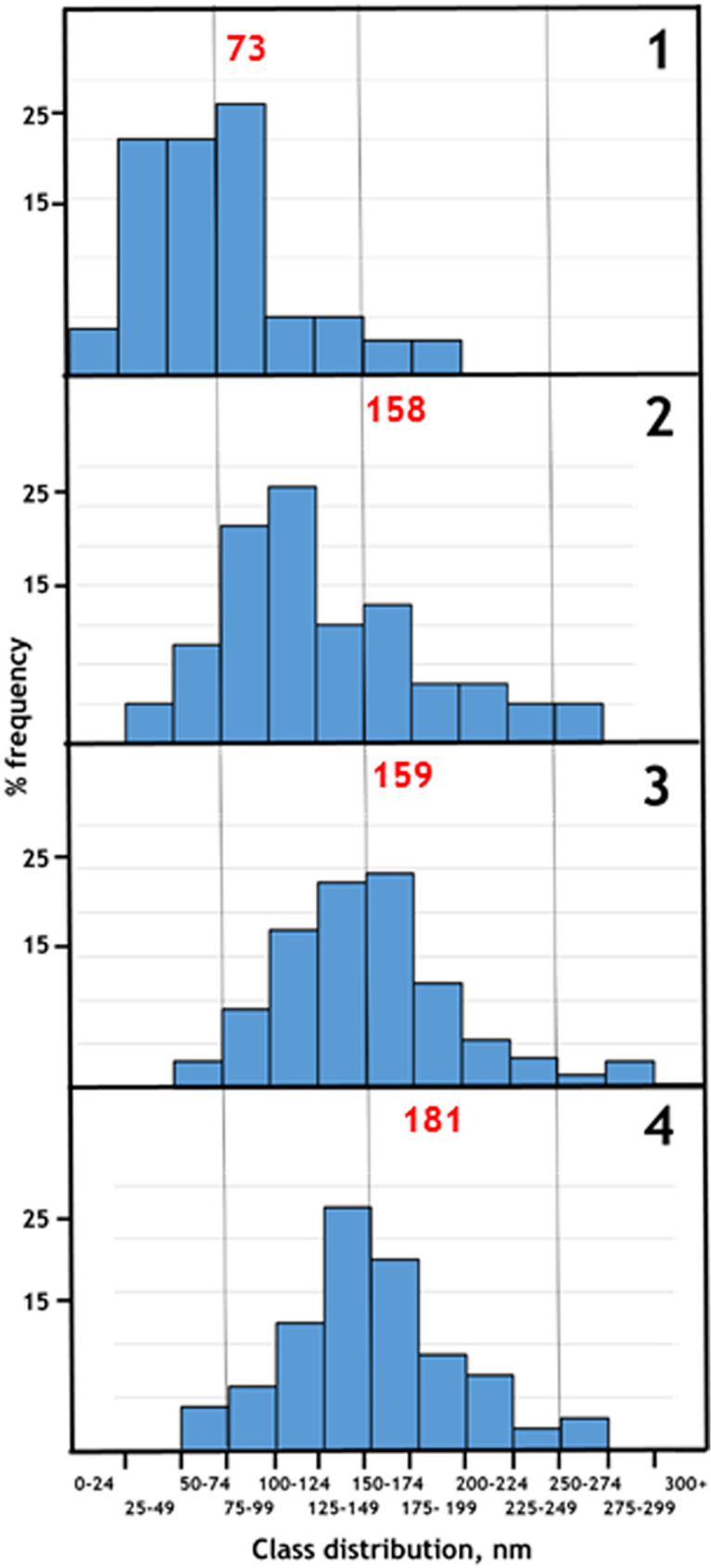

FIGURE 3 | (A) Transmission electron microscopy, and (B) distribution of the measured lengths of the generated nano-rods. Nano-rods generated from infiltrations with, 1 - pEAQ-HT-TMV CP/OAS, 2 - pEAQ-HT-TMV CPNP60/OAS, 3 - pEAQ-HT-TMV CP and pBin-KS-mim-OAS-mp del, 4 - pEAQ-HT TMV CP and pBin_CPMV-2_OAS. The length of measured nano-rods, resulting from each infiltration is indicated in (A). Scale bar = $50 \mathrm{~nm}$. Red numbers in (B) indicate the theoretical length of the nano-rods arising from each infiltration.

pEAQ-HT-TMV-CP/OAS. Furthermore, it would be difficult to produce defined length nano-rods containing several $\mathrm{CP}$ variants if both the OAS and the $\mathrm{CP}$ sequence are on the same RNA.

To investigate the possibility of in trans assembly in which the CP and the OAS are on separate RNA molecules, the OAS element was introduced into a deleted (Madi et al., 2015) and a full-length version of CPMV RNA-2 (Liu and Lomonossoff, 2002) to give constructs pBin-KS-mim-OAS-mp del and pBin_CPMV2_OAS, predicted to produce transcripts of 3392 and 3862 nucleotides, respectively, in planta, (Figure 1B). To examine the ability of these RNAs to assemble into rods, they were 
independently co-infiltrated with the pEAQ-HT-TMV CP gene construct (lacking the OAS) to create an in trans viral assembly system, (Figure 1B). Isolation and examination of the rods formed was carried out as described for the in cis system (Figure 2B, lanes 3 and 4). Negatively stained images of the particles indicated that the in trans system gave a mean rod length close to the expected length in the case of pBin-KS-mim-OAS$\mathrm{mp}$ del (152 nm vs. a calculated length of $159 \mathrm{~nm}$ ) whereas the rods produced from pBin_CPMV-2_OAS where somewhat shorter than expected (149 nm vs. $181 \mathrm{~nm}$; Figure 3, panels 3 and 4). We do not know the reason for this discrepancy but it may reflect the lack of sufficient $\mathrm{CP}$ to encapsidate the longer RNA. However, it may also be due to the fact that two gene constructs are needed for nano-rod formation with the in trans system in contrast to in cis nano-rod formation where only a single gene construct has to be present in a transformed cell. As shown previously, co-infiltration is a less efficient process than infiltration with a single construct (Montague et al., 2011). The variation of the measured length of the nano-rods produced either from single or co-infiltrations (in cis or in trans nanorod formation, respectively) in our study (Figure 3B), is in keeping with the variation measured and reported by Shukla et al. (2015) concerning the in vitro formation of nano-rods. In this previous study, in vitro synthesized RNA of a known length was incubated with purified TMV CP for up to $48 \mathrm{~h}$ to form nano-rods. Similarities in the variation of the lengths of the nanorods are apparent for both systems. Indeed the in vitro study reports that there is a greater variation in the length of the nanorods resulting from the incubation of the longest RNAs when presented to $\mathrm{CP}$, unlike the current investigation where a similar degree of variation in the length of the nano-rod is observed irrespective of its predicted length.

\section{Confirmation That Transcript Length Determines Particle Length}

When RNA was extracted from particles purified without the $\mathrm{CsCl}$ gradient step, bands corresponding to RNA of the expected length, ranging in size between 1.5 and $3.8 \mathrm{~kb}$ could be seen on denaturing agarose gels stained with ethidium bromide apart from the sample produced from plants infiltrated with pEAQ-HT-TMV-CPHis/OAS (Figure 4, lanes 1 to 5). This construct was designed to express TMV CP modified to contain six histidine residues at its C-terminus (see below); this observation suggests that this tagged $\mathrm{CP}$ is not as assembly competent as wild-type (WT) CP. In all cases the samples of RNA extracted from partially purified nano-rods also contained substantial quantities of low molecular weight nucleic acid migrating more quickly than the $0.5 \mathrm{~kb}$ marker. To determine if this material was encapsidated into nanorods or represented co-purifying host or Agrobacterium-derived nucleic acid, RNA was extracted from a sample of nanorods obtained by infiltration $\mathrm{pEAQ}-H T$-TMV-CP/OAS that had been purified from a $\mathrm{CsCl}$ gradient (Figure 4A, lanes, 6 and 7). In this case, a single band migrating close to the $1.5 \mathrm{~kb}$ marker could be seen - a size consistent with the encapsidation of the OAS-containing transcript from pEAQ$H T$-TMV-CP/OAS. Significantly none of the shorter nucleic acids banded at the density of nano-rods suggesting that they are not encapsidated but rather represent nucleic acid fragments that are co-precipitated during the PEG precipitation step.

To confirm that the high molecular weight bands seen in the ethidium bromide-stained gel (Figure 4A, lanes 1-5) represented encapsidated RNA containing the TMV OAS, a replica agarose gel was subjected to northern blot analysis using a probe specific
A

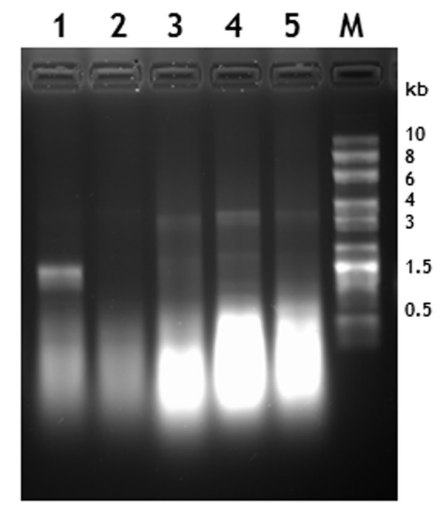

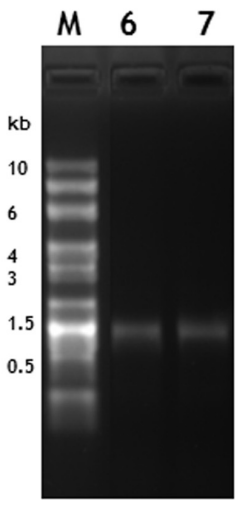

B

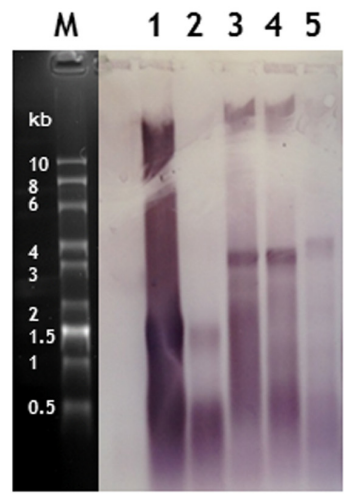

FIGURE 4 | Verification of the length and sequence of RNA molecules encapsidated in nano-rods. Denaturing agarose gels of RNA extracted from nano rods either stained with ethidium bromide (A) or blotted and probed in a northern blot with an anti-sense OAS RNA probe (B). RNA samples were extracted from the resulting nano-rods of plant infiltrations of 1- pEAQ-HT-TMV CP/OAS, 2- pEAQ-HT-TMV CPHis/OAS, 3- pEAQ-HT-TMV CPNP60/OAS, 4- pEAQ-HT TMV $\mathrm{CP}+$ pBin-KS-mim-OAS-mp del. 5- pEAQ-HT TMV CP + pBin_CPMV-2_OAS. These samples were prepared without the CHCl 3 step in the isolation procedure and the final $\mathrm{CsCl}$ gradient. Approximately $400 \mathrm{ng}$ of RNA was run in each lane for each infiltration. 6- 330 ng and 7 - $1 \mu \mathrm{g}$ of RNA extracted from nano-rods of plants infiltrated with $\mathrm{PEAQ}-\mathrm{HT}$-TMV CP/OAS and isolated with a $\mathrm{CHCl}_{3}$ step and the final $\mathrm{CsCl}$ step in the procedure. The $\mathrm{C}$ terminus of the coat protein in lane 2 contained an additional six histidine residues. The original photograph of $(\mathbf{A})$ is shown in Supplementary Figure $\mathbf{S 5}$ and similarly of (B) in Supplementary Figure S6. 
for the OAS. The specificity of the probe was confirmed by its ability to readily detect TMV RNA but not commercially available RNA size markers (Supplementary Figure S6C). In all cases, the high molecular weight RNA extracted from nano-rods hybridized with the OAS-specific probe to a degree consistent with the intensity of the ethidium bromide-stained bands; in contrast the lower molecular weight material hybridized less well, thereby suggesting that much of this material was not TMVspecific. In addition a high molecular weight band of hybridizing material could be seen in all samples (Figure 4B) which we believe represents plasmid DNA which has co-precipitated with the nano-rods. One significant difference between the results obtained by northern blotting and ethidium bromide staining was that there was evidence from the former that the RNA transcribed from pEAQ-HT-TMV-CPHis/OAS could be incorporated into nano-rods by the coat protein containing a $6 \times$ His sequence at its C-terminus albeit less efficiently that the equivalent WT construct. The results of the study of the RNA encapsidated within the nano-rods suggest rods are likely to be of a more precisely defined size than implied by the TEM analysis of the negatively stained particles. This difference is probably a reflection of the harsh nature of the TEM analysis leading to shearing of some of the particles on the grids.

\section{Incorporation of Modified Coat Proteins into Nano-Rods}

In addition to controlling particle length, another potential advantage of using transient expression to produce nano-rods is the possibility of expressing $\mathrm{CP}$ variants which may be incompatible with a productive infection. To examine this possibility, two $\mathrm{CP}$ variants with additional sequences at the C-terminus were produced, one with the addition of six histidines and the second with the sequence CNAGDHANC. The incorporation of six histidines has been shown to permit the assembly of the modified $\mathrm{CP}$ into a number of proteinonly structures but the modified protein has been reported to be poorly incorporated into RNA-containing rods (Bruckman et al., 2011; Wnek et al., 2013). The CNAGDHANC peptide was originally identified as being able to bind cobalt- platinum $(\mathrm{CoPt})$ when expressed on the surface of the coat protein of M13 bacteriophage (Mao et al., 2004) and has subsequently been used to promote the deposition of CoPt onto the surface of the spherical plant virus, cowpea mosaic virus (Aljabali et al., 2011). In both cases the sequence of the C-terminally modified $\mathrm{CP}$ was substituted for the wild-type $\mathrm{CP}$ in construct pEAQ$H T$-TMV-CP/OAS to give pEAQ-HT-TMV-CPHis/OAS and pEAQ-HT-TMV-CP(CP9)/OAS (Figure 1A). Both constructs were designed to encapsidate an RNA of $1.5 \mathrm{~kb}$ producing nanorods of around $75 \mathrm{~nm}$. pEAQ-HT-TMV-CPHis, similar in design to $\mathrm{pEAQ}-H T$-TMV-CP but possessing six C-terminal histidines, was also constructed to investigate in trans incorporation (Figure 1B).

In the case of pEAQ-HT-TMV-CPHis/OAS, the yield of nano-rods obtained after PEG precipitation of extracts from leaves infiltrated with pEAQ-HT-TMV-CPHis/OAS was about

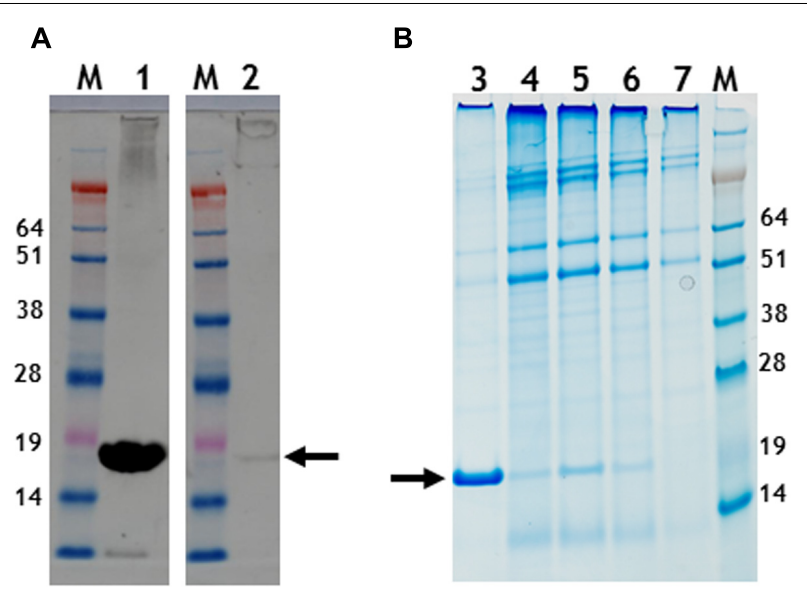

FIGURE 5 | Gel migration and western blot detection of 6 histidine C-terminal possessing nano-rods. (A) Detection of CP with a polyclonal anti-TMV antibody., Lane 1, pEAQ-HT-TMV CP/OAS and lane 2, pEAQ-HT-TMV $\mathrm{CP} / \mathrm{His} / \mathrm{OAS}$. Equal masses of leaf material was processed for each gene infiltration. (B) Instant blue staining of PEG purified nano-rods. pEAQ-HT-TMVCP/OAS, lane 3. pEAQ-HT-TMV-CPHis/OAS, lane 4. Co-infiltration of pBin-KS-mim-OAS-mp del and pEAQ-HT-TMV-CP lane 5. Co-infiltration of pBin-KS-mim-OAS-mp del and pEAQ-HT-TMV-CPHis lane 6. Co-infiltration of pBin-KS-mim-OAS-mp del, pEAQ-HT-TMV/CP and $\mathrm{pEAQ}-H T$-TMV/CPHis, lane 7. Arrows indicate the position of resolved CP. The original photographs for (A,B) are shown in Supplementary Figure S7.

$1 \%$ of that obtained from leaves infiltrated with pEAQ-HT-TMV$\mathrm{CP} / \mathrm{OAS}$, judged by the detection of CP (arrowed) by western blotting with a polyclonal antibody to TMV (Figure 5A). Here equal masses of infiltrated leaf material harvested at 7 days post infiltration from both infiltrations was processed. Instant Blue-stained gels of preparations of nano-rods isolated by PEG precipitation (Figure 5B), showed the presence of CP suggesting that the modified CP could be assembled into nano-rods via the in trans reaction, (Figure 5B, lane 6) This substantiated the finding above, concerning the detection of encapsidated RNA in the in cis derived six histidine rods by northern blotting, (Figure 4A). This was confirmed by TEM analysis which showed the presence only a few nano-rods of the expected size (data not shown). An attempt to incorporate the six histidine C-terminal $\mathrm{CP}$ into nano-rod structures via the in trans reaction upon the co-infiltration of both the WT and the six histidine CP coat protein constructs were fruitless, (Figure 5B, lane 7). This again highlights the difficulty of incorporating this modified CP into RNA containing rods, discussed above. However, the Instant Blue stained gel, (Figure 5B, lane 6), revealed the presence of CP in PEG purified nano-rods (generated via the in trans reaction). Thus, it is likely that the $\mathrm{C}$-terminal six histidines have been removed probably by protease digestion thereby allowing for the formation of WT nano-rods. Taken together, these results show that assembly of the His-tagged protein into RNA-containing nano-rods is inefficient compared to the incorporation of wild-type coat protein. This may be because of the previously noted propensity of the His-tagged coat protein to assemble into protein-only aggregates rather than nucleoprotein 

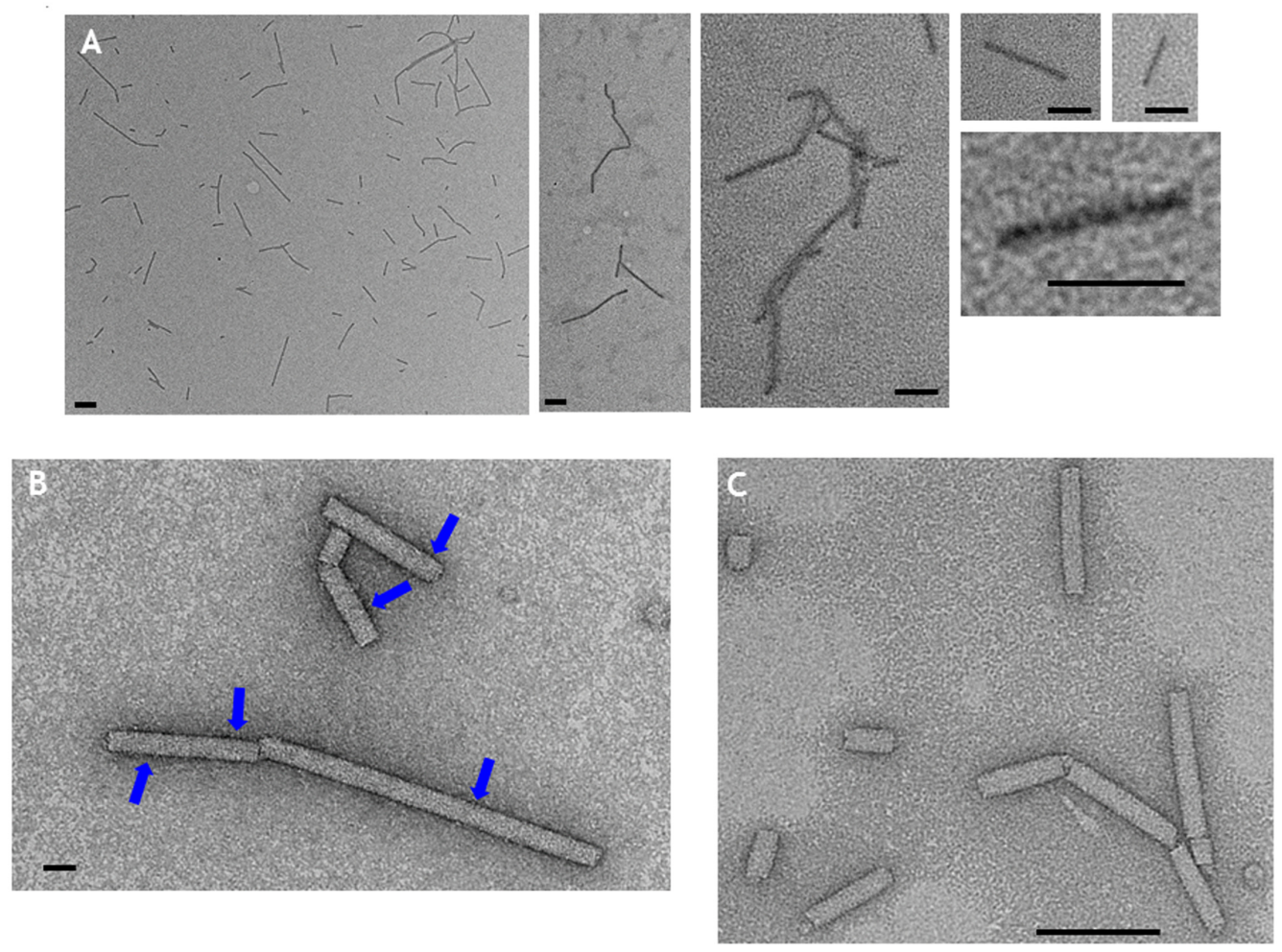

FIGURE 6 | Transmission electron microscopy of CoPt-bound CP9 nano-rods. (A) CP9 nano-rods visualized without negative staining. (B) CP9 nano-rods visualized with negative staining. (C) WT nano-rods negatively stained. Blue arrows indicate regions of the CP9 nano-rods that appear intense indicative of CoPt deposition. Scale bar; (A) $100 \mathrm{~nm}$, (B) $20 \mathrm{~nm}$, (C) $100 \mathrm{~nm}$.

rods (Bruckman et al., 2011; Wnek et al., 2013). Indeed it is possible that the small amounts of nano-rods that are observed may arise from the incorporation of subunits from which the His-tag has been proteolytically removed. In any case, the levels of nano-rods obtained were too low to permit further detailed characterization.

By contrast, extracts of leaves infiltrated with pEAQ-HTTMV-CP(CP9)/OAS appeared to contain sufficient material to allow further purification. After centrifugation of PEGprecipitated extracts from equal masses of leaves infiltrated with pEAQ-HT-TMV-CP/OAS or pEAQ-HT-TMV-CP(CP9)/OAS through $\mathrm{CsCl}$ gradients, the yield of the $\mathrm{CP} 9$ nano-rod was estimated to be approximately one third that of the WT nano-rod as judged by Instant Blue staining of coat protein resolved in NuPAGE gels (Supplementary Figure S2). CP9 nanorods were morphological indistinguishable from the WT nanorods when examined by transmission electron microscopy after negative staining with uranyl acetate (Supplementary Figure S3). Following CoPt deposition, CP9 nano-rods could be visualized without negative staining (Figure 6A). In this case, the nanorods had a dark rod-like appearance but with a hazy outline. Intense darker regions on the CP9 nano-rods can also be seen without staining, especially at higher magnifications (Figure 6A). By contrast, nano-rods produced from WT coat protein could not be visualized by transmission electron microscopy unless the samples were subjected to negative staining (Figure 6C), even after being subjected to the CoPt deposition reaction. Negative staining of the CP9 nano-rods following CoPt deposition revealed the characteristic nano-rod structure (Figure 6B). The build-up of density, on the surface of the CP9 nano-rods is also evident (Figure 6B; blue arrows) which correlates with the darker regions observed on the unstained CP9 nano-rods (Figure 6A). These darker regions were more evident in the samples which underwent a longer deposition reaction (69 h) compared with those which were reacted for a shorter time, (22 h).

In this paper we have demonstrated that it is possible to synthesize defined length nano-rods by transiently expressing the TMV coat protein and an RNA containing the OAS element. These designer nano-rods have the general morphology of native TMV rods whose length can be controlled. In addition, they will lack the ability to cause an infection since the genomic RNA is not present. The ability to control their length, makes these synthetic nano-rods ideal for many emerging nanobiotechnological applications. In recent times, the concept of using plants as biofactories for the development and production of suitable pharmaceutical proteins has been gaining strength and is now very much a commercial reality (Lomonossoff and D'Aoust, 2016). With such facilities, any problematical low yielding but modified nano-rods can easily be overcome, simply by increasing the efficiency of production. As we have demonstrated by genetic modification to the coat protein 
outer surface, it is possible to direct the synthesis of "mutant" nano-rods whose surface properties will allow for them to bind metals or functional proteins. Though we have demonstrated the production of linear rods, further applications are possible. For example, the in vitro formation of nanoboomerangs and tetrapods, formed upon RNAs with more than one OAS element has been demonstrated (Eber et al., 2015) and we anticipate that similar structures could be synthesized transiently in plants following the procedures described in this paper. In addition it is possible to incorporate modified versions of the TMV coat protein into rods of predetermined size without the need either for preserving viral infectivity or having to undertake in vitro reassembly experiments. The ability to metallise rods of different sizes, as demonstrated in the case of CoPt, increases the scope for the deployment of TMV-based structures in nanoelectronics.

\section{AUTHOR CONTRIBUTIONS}

Conceived and designed the experiments: KS and GL. Performed the experiments: KS. Analyzed the data: KS and GL. Wrote the paper: KS and GL.

\section{FUNDING}

The research was supported by the United Kingdom Biotechnological and Biological Sciences Research Council (BBSRC) Institute Strategic Programme Grant 'Understanding and Exploiting Plant and Microbial Secondary Metabolism' (BB/J004596/1) and the John Innes Foundation.

\section{ACKNOWLEDGMENTS}

We thank Elaine Barclay for help with TEM and the measurement of many nano-rods, Gerhard Saalbach for MALDI-TOF analysis,

\section{REFERENCES}

Aljabali, A. A. A., Shah, S. N., Evans-Gowing, R., Lomonossoff, G. P., and Evans, D. J. (2011). Chemically-coupled-peptide-promoted virus nanoparticle templated mineralization. Integr. Biol. 3, 119-125. doi: 10.1039/c0ib0 $0056 \mathrm{f}$

Bruckman, M. A., Soto, C. M., McDowell, H., Liu, J. L., Ratna, B. R., Korpany, K. V., et al. (2011). Role of hexahistidine in directed nanoassemblies of tobacco mosaic virus coat protein. ACS Nano 5:1606. doi: 10.1021/nn1025719

Butler, P. J. G. (1984). The current picture of the structure and assembly of tobacco mosaic virus. J. Gen. Virol. 65, 253-279. doi: 10.1099/0022-1317-65-2-253

Butler, P. J. G., Finch, J. T., and Zimmern, D. (1977). Configuration of tobacco mosaic virus RNA during virus assembly. Nature 265, 217-219. doi: 10.1038/ $265217 \mathrm{a} 0$

Butler, P. J. G., and Klug, A. (1971). Assembly of the particle of tobacco mosaic virus from RNA and disks of protein. Nat. New Biol. 229, 47-50. doi: 10.1038/ newbio229047a0

Eber, F. J., Eiben, S., Jeske, H., and Wege, C. (2015). RNA-controlled assembly of tobacco mosaic virus-derived complex structures: from nanoboomeranges to tetrapods. Nanoscale 7, 344-355. doi: 10.1039/c4nr05434b
David Evans and Martin Rejzek for discussions about metal binding and Andrew Davis for photography.

\section{SUPPLEMENTARY MATERIAL}

The Supplementary Material for this article can be found online at: http://journal.frontiersin.org/article/10.3389/fpls.2017.01335/ full\#supplementary-material

FIGURE S1 | (A) Nucelotide sequence of the synthetic DNA that was cloned into pEAQ-HT to yield pEAQ-HT-TMV-CP/OAS. (B) Nucleotide sequence of the synthetic DNA that was cloned into $P E A Q-H T$ to yield PEAQ-HT-TMV-CP(CP9)/OAS. The relevant restriction sites used in this cloning and subsequent DNA manipulations are highlighted in bold text.

FIGURE S2 | Separation of TMV, WT, and CP9 nano-rods by centrifugation in $10-50 \%$ step sucrose gradient. Samples were spun at $145,000 \times g$ for $1 \mathrm{~h}$ at $5^{\circ} \mathrm{C}$ and fractionated into $300 \mu \mathrm{L}$ fractions and $10 \mu \mathrm{L}$ of each fraction was resolved on $12 \%$ NuPAGE gels, with the top fraction of the gradient on the left of each gel. TMV CP was visualized by Instant Blue staining. (A) TMV; (B) CP9 nano-rods; and (C) WT nano-rods.

FIGURE S3 | Visualization of UA stained nano-rod structures. (A) TMV; (B) CP9 nano-rods; and (C) WT nano-rods by transmission electron microscopy.

FIGURE S4 | Un-edited photograph of $\mathrm{CsCl}$ gradients. A cut down portion, tube 3 was used in Figure 2A. Un-edited photographs of two original gels used to make Figure 2B. The original positions of lanes $1-4$ and $M$ are indicated.

FIGURE S5 | Un-edited photograph of the ethidium bromide stained gel used in Figure 4A. Used lanes, displayed in Figure $\mathbf{4}$ are indicated. Portions between them were removed for the final figure.

FIGURE S6 | (A) Original photograph of ethidium bromide-stained gel showing the RNA size markers (Invitrogen, catalog number 15623-200) run (Lane M) with samples that were subsequently transferred to a nylon membrane to create Figure 4B. (B) Original photograph of the northern blot, lanes 1-5, displayed in Figure 4B. (C) Denaturing agarose gel of Ambion Millenium RNA Markers, catalog number AM 7150 (Lane 1) and $1 \mu \mathrm{g}$ TMV RNA (lane 2) stained with ethidium bromide (right) and subsequently subjected to northern blot analysis using the OAS-specific probe (left). Only the TMV RNA can be detected with the probe.

FIGURE S7 | (A) Original photograph of the anti TMV western blot used in Figure 5A. (B) Instant blue NuPAGE stained gel. Used lanes for Figure $\mathbf{5}$ are indicated.

Fan, X. Z., Pomerantseva, E., Gnerlich, M., Brown, A., Gerasopoulos, K., McCarthy, M., et al. (2013). Tobacco mosaic virus: a biological building block for micro/nano/bio systems. J. Vac. Sci. Technol. A 31, 050815. doi: 10.1116/1. 4816584

Fraenkel-Conrat, H., and Williams, R. C. (1955). Reconstitution of active tobacco mosaic virus from its inactive protein and nucleic acid components. Proc. Natl. Acad. Sci. U.S.A. 41, 690-698. doi: 10.1073/pnas.41.10.690

Gallie, D. R., Plaskitt, K. A., and Wilson, T. M. A. (1987). The effect of multiple dispersed copies of the origin-of-assembly sequence from TMV RNA on the morphology of pseudovirus particles assembled in vitro. Virology 158, 473-476. doi: 10.1016/0042-6822(87)90225-X

Goelet, P., Lomonossoff, G. P., Butler, P. J. G., Akam, M. E., Gait, M. J., and Karn, J. (1982). Nucleotide sequence of tobacco mosaic virus RNA. Proc. Natl. Acad. Sci. U.S.A. 79, 5818-5822. doi: 10.1073/pnas.79.19.5818

Hwang, D. J., Roberts, I. M., and Wilson, T. M. A. (1994). Expression of tobacco mosaic virus coat protein and assembly of pseudovirus particles in Escherichia coli. Proc. Natl. Acad. Sci. U.S.A. 91, 9067-9071. doi: 10.1073/pnas.91.19.9067

Kadri, A., Wege, C., and Jeske, H. (2013). In vivo self-assembly of TMV-like particles in yeast and bacteria for nanotechnological applications. J. Virol. Methods 189, 328-340. doi: 10.1016/j.jviromet.2013.02.017 
King, D. P., Montague, N., Ebert, K., Dukes, J. P., Schädlich, L., Belsham, G. J., et al. (2007). Development of a novel recombinant encapsidated RNA particle: evaluation as an internal control for diagnostic RT-PCR. J. Virol. Methods 146, 218-225. doi: 10.1016/j.jviromet.2007.07.002

Liu, L., and Lomonossoff, G. P. (2002). Agroinfection as a rapid method for propagating Cowpea mosaic virus-based constructs. J. Virol. Methods 105, 343-348. doi: 10.1016/S0166-0934(02)00121-0

Lomonossoff, G. P., and Butler, P. J. G. (1979). Location and encapsidation of the coat protein cistron of tobacco mosaic virus. Eur. J. Biochem. 93, 157-164.

Lomonossoff, G. P., and Butler, P. J. G. (1980). Assembly of tobacco mosaic virus: elongation towards the 3' hydroxyl terminus of the RNA. FEBS Lett. 113, 271-274. doi: 10.1016/0014-5793(80)80607-7

Lomonossoff, G. P., and D'Aoust, M.-A. (2016). Plant-produced biopharmaceuticals: a case of technical developments driving clinical development. Science 353, 1237-1240. doi: 10.1126/science.aaf6638

Lomonossoff, G. P., and Evans, D. J. (2011). "Applications of plant viruses in bionanotechnology," in Current Topics of Microbiology and Immunology, Vol. 375, eds K. Palmer and Y. Gleba (Berlin: Springer), 61-87. doi: 10.1007/82_ 2011_184

Madi, M., Mioulet, V., King, D. P., Lomonossoff, G. P., and Montague, N. (2015). Development of a non-infectious encapsidated positive control RNA for molecular assays to detect foot-and-mouth disease virus. J. Virol. Methods 15, 27-34. doi: 10.1016/j.jviromet.2015.04.002

Mao, C., Solis, D. J., Reiss, B. D., Kottmann, S. T., Sweeney, R. Y., Hayhurst, A., et al. (2004). Virus-based toolkit for the directed synthesis of magnetic and semiconducting nanowires. Science 303, 213-217. doi: 10.1126/science.1092740

Meltzer, J. C., Sanders, V., Grimm, P. C., Chiasson, N., Hoeltke, H.-J., Garrett, K. L., et al. (1998). Nonradioactive northern blotting with biotinylated and digoxigenin-labeled RNA probes. Electrophoresis 19, 1351-1355. doi: 10.1002/ elps. 1150190825

Meshcheriakova, Y., Saxena, P., and Lomonossoff, G. P. (2014). Fine-tuning levels of heterologous gene expression in plants by orthogonal variation of the untranslated regions of a nonreplicating transient expression system. Plant Biotechnol. J. 12, 718-727. doi: 10.1111/pbi.12175

Montague, N. P., Thuenemann, E. C., Saxena, P., Saunders, K., Lenzi, P., and Lomonossoff, G. P. (2011). Recent advances of cowpea mosaic virus-based particle technology. Hum. Vacc. 7, 383-390. doi: 10.4161/hv.7.3.14989

Sainsbury, F., and Lomonossoff, G. P. (2008). Extremely high-level and rapid transient protein production in plants without the use of viral replication. Plant Physiol. 148, 1212-1218. doi: 10.1104/pp.108.126284

Sainsbury, F., Saxena, P., Aljabali, A. A. A., Saunders, K., Evans, D. J., and Lomonossoff, G. P. (2014). "Genetic engineering and characterization of cowpea Mosaic Virus Empty virus-like particles," in Virus Hybrids as Nanomaterials: Methods and Protocols, Methods in Molecular Biology, Vol. 1108, eds B. Lin and B. Ratna (New York, NY: Springer Science+Business Media). doi: 10.1007/978-1-62703-751-8_11

Sainsbury, F., Thuenemann, E. C., and Lomonossoff, G. P. (2009). pEAQ: versatile expression vectors for easy and quick transient expression of heterologous proteins in plants. Plant Biotechnol. J. 7, 682-693. doi: 10.1111/j.1467-7652. 2009.00434.x

Saunders, K., and Lomonossoff, G. P. (2015). The generation of turnip crinkle virus-like particles in plants by the transient expression of wild-type and modified forms of its coat protein. Front. Plant Sci. 6:1138. doi: 10.3389/fpls. 2015.01138

Shire, S. J., McKay, P., Leung, D. W., Cachianes, G. J., Jackson, E., Wood, W. I., et al. (1990). Preparation and properties of recombinant DNA derived tobacco mosaic virus coat protein. Biochemistry 29, 5119-5126. doi: 10.1021/ bi00473a017

Shukla, S., Eber, F. J., Nagarajan, A. S., DiFranco, N. A., Schmidt, N., Wen, A. M., et al. (2015). The impact of aspect ratio on the biodistribution and tumor homing of rigid soft-matter nanorods. Adv. Healthcare Mater. 4, 874-882. doi: 10.1002/adhm.201400641

Sleat, D. E., Gallie, D. R., Watts, J. W., Deom, C. M., Turner, P. C., Beachy, R. N., et al. (1988). Selective recovery of foreign gene transcripts as virus-like particles in TMV-infected transgenic tobaccos. Nucleic Acids Res. 16, 3127-3140. doi: 10.1093/nar/16.8.3127

Sleat, D. E., Turner, P. C., Finch, J. T., Butler, P. J., and Wilson, T. M. A. (1986). Packaging of recombinant RNA molecules into pseudovirus particles directed by the origin-of-assembly sequence from tobacco mosaic virus RNA. Virology 155, 299-308. doi: 10.1016/0042-6822(86) 90194-7

Turner, D. R., McGuigan, C. J., and Butler, P. J. G. (1989). Assembly of hybrid RNAs with tobacco mosaic virus coat protein. Evidence for incorporation of disks in 5'-elongation along the major RNA tail. J. Mol. Biol. 209, 407-422. doi: 10.1016/0022-2836(89)90006-5

Wnek, M., Gorzny, Ł., Ward, M. B., Walti, C., Davies, A. G., Brydson, R., et al. (2013). Fabrication and characterization of gold nano-wires templated on viruslike arrays of tobacco mosaic virus coat proteins. Nanotechnology 24:025605. doi: 10.1088/0957-4484/24/2/025605

Zimmern, D. (1977). The nucleotide sequence at the origin for assembly on tobacco mosaic virus RNA. Cell 11, 463-482. doi: 10.1016/0092-8674(77) 90065-4

Zimmern, D., and Butler, P. J. G. (1977). The isolation of tobacco mosaic virus RNA fragments containing the origin for viral assembly. Cell 11, 455-462. doi: 10.1016/0092-8674(77)90064-2

Zimmern, D., and Wilson, T. M. A. (1976). Location of the origin for viral reassembly on tobacco mosaic virus RNA and its relation to stable fragment. FEBS Lett. 71, 294-298. doi: 10.1016/0014-5793(76) 80954-4

Conflict of Interest Statement: GL declares that he is a named inventor on granted patent WO 29087391 A1 which describes the $\mathrm{pEAQ}$ vector system used for the work described in this manuscript.

The other author declares that the research was conducted in the absence of any commercial or financial relationships that could be construed as a potential conflict of interest.

Copyright (c) 2017 Saunders and Lomonossoff. This is an open-access article distributed under the terms of the Creative Commons Attribution License (CC BY). The use, distribution or reproduction in other forums is permitted, provided the original author(s) or licensor are credited and that the original publication in this journal is cited, in accordance with accepted academic practice. No use, distribution or reproduction is permitted which does not comply with these terms. 